\title{
Photon Echoes from Semiconductor Band-to-Band Continuum Transitions in the Regime of Coulomb Quantum Kinetics
}

\author{
W. A. Hügel, M. F. Heinrich, and M. Wegener \\ Institut für Angewandte Physik, Universität Karlsruhe (TH), Kaiserstraße 12, D-76128 Karlsruhe, Germany \\ Q. T. Vu, L. Bányai, and H. Haug \\ Institut für Theoretische Physik, J. W. Goethe-Universität Frankfurt, Robert-Mayer-Strasse 8, \\ D-60054 Frankfurt am Main, Germany
}

(Received 29 January 1999)

\begin{abstract}
We show that the coherent response of semiconductor band-to-band continuum transitions at room temperature, excited by 11 fs pulses at elevated carrier densities, deviates substantially from that expected for an ideal photon echo. A microscopic non-Markovian quantum kinetic theory of both electron-electron and electron-LO-phonon scattering reproduces these unusual experimental observations qualitatively and, furthermore, the famous scaling of the decay time $\tau$ with carrier density $n_{\mathrm{eh}}$ according to $\tau^{-1}=\tau_{0}^{-1}+c n_{\mathrm{eh}}^{1 / 3}$ quantitatively. $\tau$, however, cannot be associated with a single unique phase relaxation time $T_{2}$.

PACS numbers: 78.47.+p, 42.50.Md, 42.65.Re, 63.20.Kr
\end{abstract}

Consider a dense gas of electrons and holes generated at random places in pairs with opposite momentum in coherent quantum states in a semiconductor. It is clear that the particles will move in such a way that they screen their mutual Coulomb interaction. How long does it take that screening is built up and that scattering has destroyed the initially quantum mechanically coherent state? About one decade ago, Shank et al. addressed this fundamental question when they studied the variation of the Coulomb scattering in a dense gas of optically excited crystal electrons and holes [1,2] with density $n_{\mathrm{eh}}$ using $\approx 10 \mathrm{fs}$ pulses and a photon echo technique. They reported that the decay time $\tau$ of the photon echo signal at room temperature scales approximately according to $\tau \propto n_{\mathrm{eh}}^{-1 / D}$, where $D$ is the dimension of either bulk GaAs $(D=3)$ or GaAs quantum wells $(D=2)$. At that time, it was tempting and appealing to interpret this seemingly simple result in terms of the nearest-neighbor scattering of $D$-dimensional hard spheres in which case it is simple to show that the average time between different collisions scales $\propto n_{\mathrm{eh}}^{-1 / D}$ indeed.

Today, a number of important questions are still unanswered. (i) Is it a photon echo? In analogy with a spin echo this would mean that a first short pulse excites the system, a second pulse arrives after time delay $t_{21}$, and the coherent collective response of the system, the photon echo, peaks at yet another time delay $t_{21}$ later. Without real-time resolution $[1,2]$, however, one only measures the energy of the echo signal versus $t_{21}$ and its decay time $\tau$. For a phenomenological dephasing time $T_{2}$ it is known that $T_{2}=4 \tau$ holds for a four-wave mixing (FWM) geometry under these conditions. Not a single experiment, however, has shown directly that the coherent real-time response of semiconductor band-to-band continuum transitions under these conditions is a photon echo indeed. Under quite dif- ferent conditions ( $T=4 \mathrm{~K}, 100$ fs pulses, and much lower carrier densities), Ref. [3] showed that the total response is not a photon echo. Only after spectral filtering (behind the sample) of those components of the FWM-signal resonant with the band-to-band continuum, were they able to isolate a contribution which corresponded to an echo. The position of this signal shifted with time delay as expected [4], its width was constant (150 fs) and given by the time resolution of their setup for all conditions. The picosecond response of inhomogeneously broadened excitonic transitions [5] as well as the response of modulation-doped quantum wells [6] followed the expectation for an ideal photon echo. Other real-time resolved work has addressed the coherent response of the homogeneously broadened $1 \mathrm{~s}$ exciton [7,8]. (ii) Can the absolute values of the measured decay time $\tau$ and their scaling with carrier density $n_{\text {eh }}$ be explained quantitatively by theory? So far the answer has been no. Such a theory of electron-electron scattering has to account for the fact that the excited carriers initially behave as coherent quantum mechanical waves. In other words: This theory has to account for the finite duration of scattering processes. Also, such a theory has to include the kinetics of the buildup of screening. This means that the range of the screened Coulomb potential at time $t$ does not only depend on the carrier distribution functions at that same time $t$ but also on their history. The recently developed quantum kinetic theory of electron-electron scattering [9] accounts for these aspects in analogy to our previous work on electron-phonon scattering $[10,11]$. (iii) Is the frequently used concept of a dephasing time $T_{2}$ meaningful under these conditions at all? If the memory effects of screening and scattering discussed in (ii) are really important, the $T_{2}$ concept breaks down.

In this Letter, we first reproduce the basic observations of Shank et al. concerning the time-integrated FWM on 
bulk GaAs. By additional real-time resolved experiments we address the above questions (i)-(iii). This sheds new light upon the interpretation of the famous $\tau^{-1}=\tau_{0}^{-1}+c n_{\mathrm{eh}}^{1 / 3}$ law.

Description of experiment. - Two colinearly polarized pulses, 1 and 2, arriving at times $t=t_{1}$ and $t=t_{2}$ and propagating with wave vectors $\vec{q}_{1}$ and $\vec{q}_{2}$, respectively, interact in the sample and lead to a diffracted FWM signal with wave vector $2 \vec{q}_{2}-\vec{q}_{1}$ which is monitored as a function of time delay $t_{21}=t_{2}-t_{1}$ and the realtime $t$. For clarity, we use the convention $t_{2}=0$. Thus, for a photon echo situation, we expect a signal maximum at time $t=t_{21}$. On the other hand, a free polarization decay would not shift with time delay $t_{21}$ but rather peak at a fixed point in time $t$. Using an up-conversion [6] technique with nearly transform-limited $11 \mathrm{fs}, \operatorname{sech}^{2}(t)$ shaped optical pulses, we measure a time resolution of $5 \mathrm{fs}$ (both, for the $t_{21}$ and for the $t$ domain). The error in the determination of the absolute positions $t=0$ and $t_{21}=0$ via scattered laser light is $\pm 10 \mathrm{fs}$. The electronhole pair densities $n_{\mathrm{eh}}$ which are quoted below are always defined as the sum of the two individual one-pulse

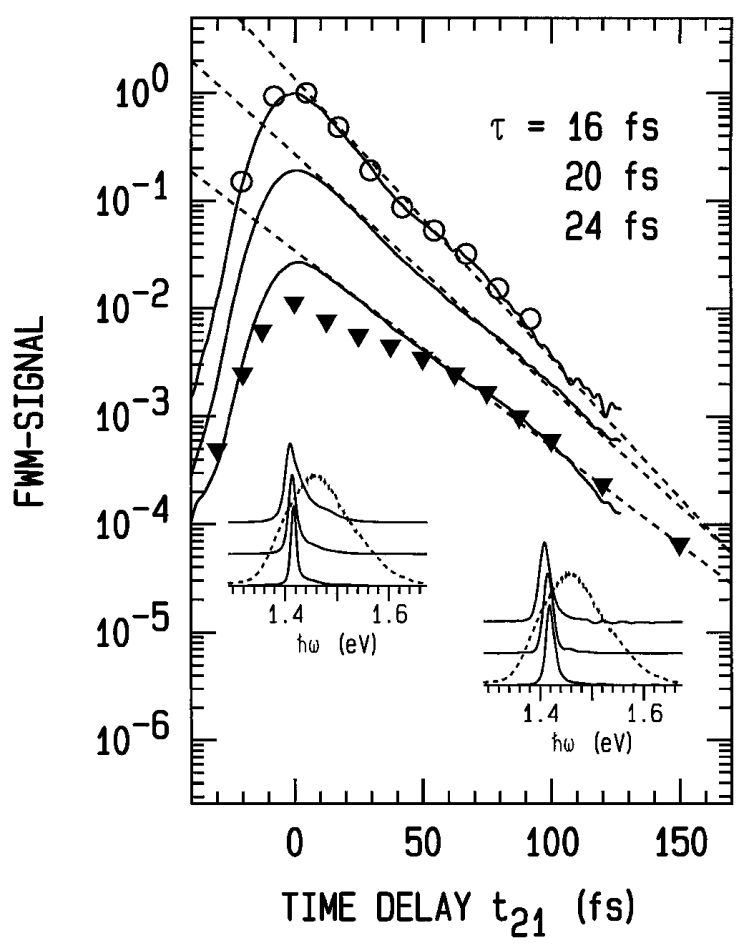

FIG. 1. Time-integrated FWM signal versus time delay for three different optically excited carrier densities $n_{\mathrm{eh}}=0.5,1$, and $2 \times 10^{17} \mathrm{~cm}^{-3}$ (from bottom to top). The full triangles are the result of the quantum kinetic theory for $n_{\mathrm{eh}}=0.57 \times$ $10^{17} \mathrm{~cm}^{-3}$. The open circles are the time-integrated data of Fig. 2, shifted by 8 fs. The decay time constants $\tau$ correspond to the dashed straight lines. The left (right) inset shows the vertically displaced and normalized FWM spectra (linear scale) for $t_{21}=0\left(t_{21}=100 \mathrm{fs}\right)$ and the laser spectrum (dashed). Bulk GaAs, $T=300 \mathrm{~K}$, excitation with 11 fs optical pulses centered $50 \mathrm{meV}$ above the band edge. carrier densities, i.e., as $n_{\mathrm{eh}}=n_{\mathrm{eh}}^{1}+n_{\mathrm{eh}}^{2}$ with $n_{\mathrm{eh}}^{2} / n_{\mathrm{eh}}^{1}=$ 5. The sample is a high quality $\mathrm{GaAs} / \mathrm{Al}_{0.3} \mathrm{Ga}_{0.7} \mathrm{As}$ double heterostructure. It contains a $0.6 \mu \mathrm{m}$ thin layer of GaAs and is glued to a sapphire disk on one side and is antireflection coated on the other side. From the measured exciton linewidth of this GaAs sample [10,11], we can safely rule out that any undesired inhomogeneous broadening is relevant for the present experiments under room temperature conditions.

Experimental results. - First, we relate our experiment to that of Ref. [1]. Figure 1 shows the time-integrated FWM signal versus time delay for three different carrier densities on the same logarithmic scale. Beside minor structures, the FWM traces decay with exponential time constants $\tau$ as indicated by the dashed straight lines in Fig. 1. ( $\tau$ vs $n_{\text {eh }}$ is shown in Fig. 5 and will be discussed later.) Selected spectra of the FWM signal at $t_{21}=0$ and $t_{21}=100 \mathrm{fs}$, respectively, are shown as insets in Fig. 1. The dashed curve is the laser spectrum. Note that the FWM spectrum at $t_{21}=0$ becomes broader with increasing $n_{\mathrm{eh}}$, which indicates that different distributions of optical continuum transitions contribute to the FWM signal. Hence, for different $n_{\mathrm{eh}}, \tau$ stems from different averages. As an example of the measured real-time responses, Fig. 2 shows the data corresponding to the conditions in

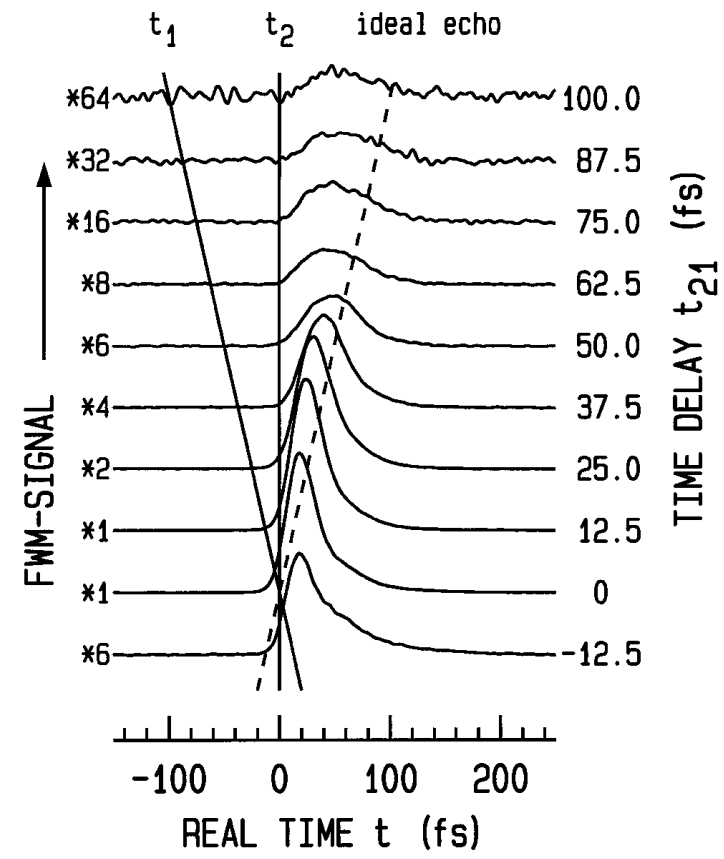

FIG. 2. Real-time $(t)$ resolved FWM signal (linear scale) for the same conditions as in Fig. $1\left(n_{\mathrm{eh}}=2 \times 10^{17} \mathrm{~cm}^{-3}\right)$. The curves are vertically displaced, the corresponding time delay $t_{21}$ can be seen on the right-hand side. The scaling factors of the various curves are different as indicated on the lefthand side for each curve. The full straight lines correspond to the temporal position of the two incident pulses, arriving at times $t=t_{1}$ and $t=t_{2}=0$, respectively; the dashed straight line is the expected position for an ideal photon echo, i.e., $t=t_{21}=t_{2}-t_{1}=-t_{1}$. 
Fig. $1\left(n_{\mathrm{eh}}=2 \times 10^{17} \mathrm{~cm}^{-3}\right)$. The real-time response is a single-side exponential with rise-time constant $<5$ fs for $t_{21}<0$. For $t_{21}>0$, the signal acquires a more symmetric shape. The position versus time delay roughly follows the ideal photon echo up to $t_{21}=50 \mathrm{fs}$. For larger time delays, the position of the echo is roughly constant again, hence, the actual echo comes earlier than expected for an ideal echo. The position and the width (full width at half maximum, FWHM) of the echo can be seen more clearly in Fig. 3, where the data of Fig. 2 and that of two other carrier densities are analyzed. Note the monotonous rise of the echo width which amounts to more than a factor of 2. This rise is consistent with the fact that the FWM spectra in Fig. 1 become narrower for larger time delays $t_{21}$ as compared to $t_{21}=0$. A single unique $T_{2}$ cannot explain these observations [4], neither can a wave vector-dependent $T_{2}$ [6]. This already suggests that the deviations from an ideal photon echo response observed here are due to many-body correlations, and, in particular, that they are specific for the regime of Coulomb quantum kinetics. We now show that the experimental evidence can be reproduced by a quantum kinetic theory of Coulombic electron-electron scattering indeed.

Description of theory. - In the framework of Keldysh nonequilibrium Green functions (GF) [12], the scattering self-energies are taken in the so-called $G W$ approximation, i.e., $\sum_{\vec{k}}\left(t_{1}, t_{2}\right)=i \sum_{\vec{q}} G_{\vec{k}-\vec{q}}\left(t_{1}, t_{2}\right) W_{\vec{q}}\left(t_{1}, t_{2}\right)$. The time-

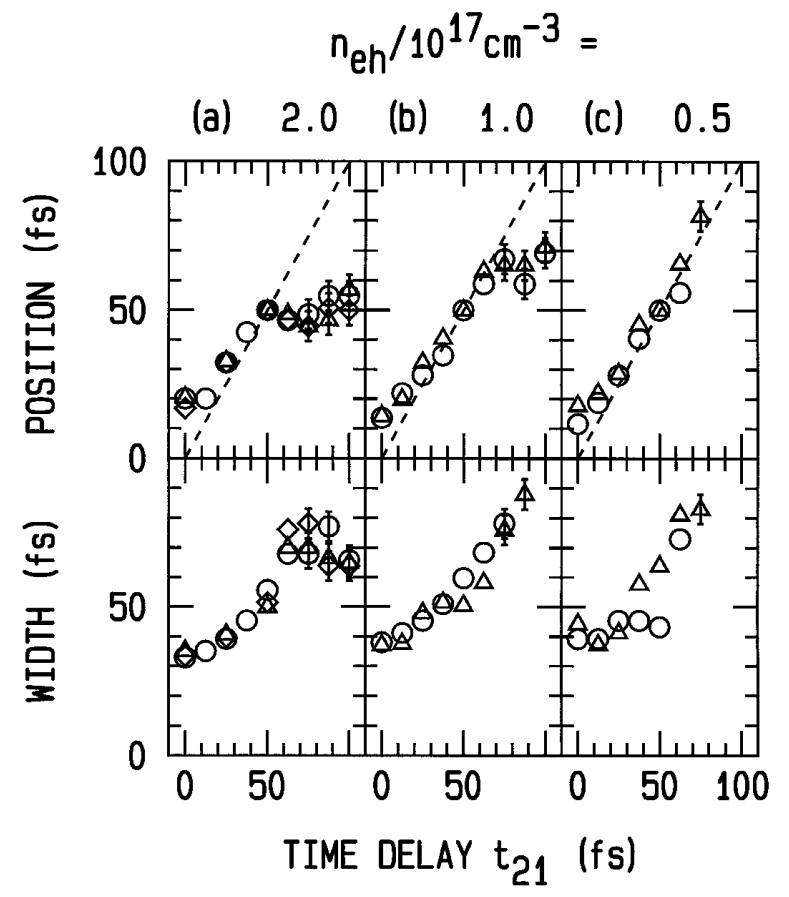

FIG. 3. The position and the width (full width at half maximum) of the photon echo signal versus time delay $t_{21}$ as extracted from the data shown in Fig. 2 and others. The dashed straight lines correspond to the expected temporal position $t$ for an ideal photon echo, i.e., to $t=t_{21}$. The width of the echo would be constant for an ideal echo. dependent screened Coulomb potential $W_{\vec{q}}$ is determined self-consistently as the solution of the integral equation $W_{\vec{q}}\left(t_{1}, t_{2}\right)=V_{\vec{q}} \delta\left(t_{1}-t_{2}\right)+V_{\vec{q}} L_{\vec{q}}\left(t_{1}, t_{3}\right) W_{\vec{q}}\left(t_{3}, t_{2}\right)$. Here a matrix notation is used for the contour time arguments. $L_{\vec{q}}\left(t_{1}, t_{2}\right)$ is the generalized random-phaseapproximation (RPA) polarization function, which includes also off-diagonal elements of the GF. These describe band mixing and excitonic effects in the coherently excited crystal. In this approach the potential $W_{\vec{q}}$ evolves from the originally unscreened potential $V_{\vec{q}}$ to a dynamically screened potential after times longer than the inverse plasma frequency of the excited carriers. Recently, we succeeded in solving the semiconductor Bloch equations together with the delayed quantum kinetics scattering integrals on conventional workstations [9]. Under the present experimental conditions, electron-electron scattering dominates over electron-phonon scattering. For completeness, however, the quantum kinetics of electronLO-phonon scattering at $T=300 \mathrm{~K}$ is also accounted for $[10,13]$. In order not to overburden the numerical calculation, we describe the corresponding spectral functions, which determine the memory kernels of the scattering integrals, by a free-particle Wigner-Weisskopf approximation [14]. We use the two-band effective mass approximation with GaAs parameters, i.e., an electron/ hole mass ratio of $m_{\mathrm{e}} / m_{\mathrm{h}}=1 / 6.7$. $11 \mathrm{fs} \operatorname{sech}^{2}(t)$ pulses with $50 \mathrm{meV}$ excess energy above the band gap excite a

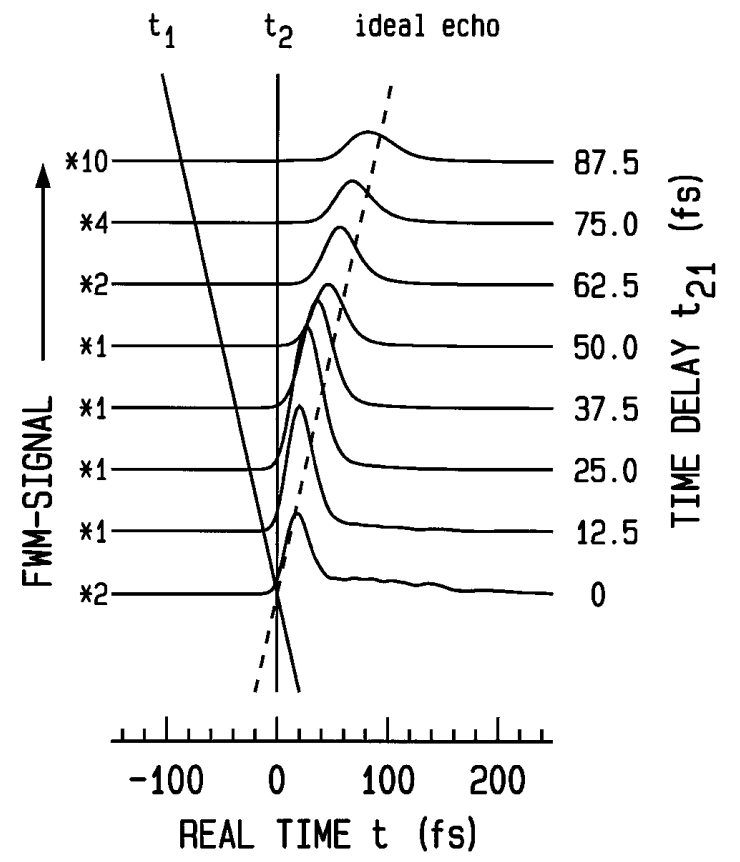

FIG. 4. Coulomb quantum kinetic theory: Real-time $(t)$ resolved FWM signal (linear scale), convoluted with an $11 \mathrm{fs}$ $\operatorname{sech}^{2}(t)$ pulse to model the up-conversion process. These curves can be compared with the experimental data shown in Fig. 2. $n_{\mathrm{eh}}=2.2 \times 10^{17} \mathrm{~cm}^{-3}$, bulk GaAs parameters, $T=$ $300 \mathrm{~K}$, excitation with $11 \mathrm{fs}$ optical pulses centered $50 \mathrm{meV}$ above the band edge. 


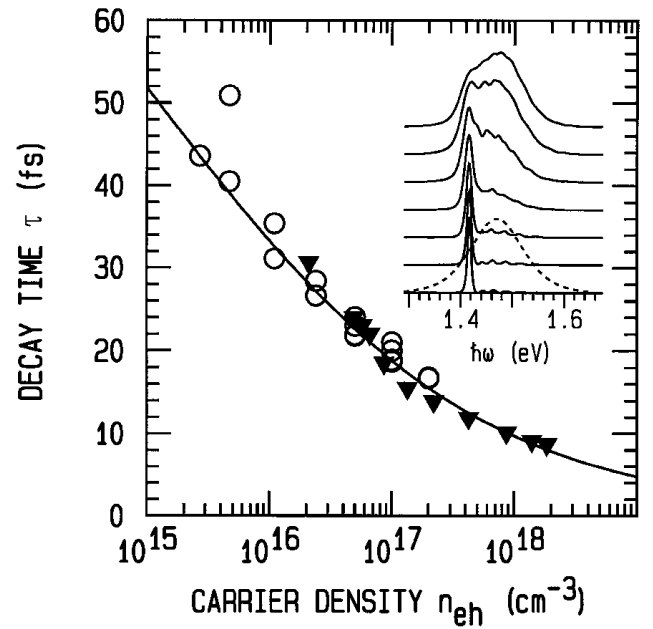

FIG. 5. Decay time $\tau$ of the time-integrated FWM signal versus carrier density $n_{\mathrm{eh}}$. The experimental data (open circles) and the result of the quantum kinetic theory (full triangles) are compared with a simple fit according to $\tau^{-1}=\tau_{0}^{-1}+c n_{\mathrm{eh}}^{1 / 3}$ with $\tau_{0}=100$ fs. The inset shows the computed FWM spectra (linear scale, normalized and vertically displaced) at $t_{21}=0$ for carrier densities $n_{\mathrm{eh}}: 0.86,1.34,2.2,4.2,8.66,13.9$, and $18.4 \times 10^{17} \mathrm{~cm}^{-3}$ (from bottom to top). The dashed curve is the laser spectrum.

total carrier density which is defined as in the experiment, i.e., according to $n_{\mathrm{eh}}=n_{\mathrm{eh}}^{1}+n_{\mathrm{eh}}^{2}$ with $n_{\mathrm{eh}}^{2} / n_{\mathrm{eh}}^{1}=5$. The FWM signal propagating with wave vector $2 \vec{q}_{2}-\vec{q}_{1}$ is extracted by a projection procedure described in Ref. [10].

Theoretical results. - Figure 4 shows the FWM signal computed this way for a total carrier density of $n_{\mathrm{eh}}=$ $2.2 \times 10^{17} \mathrm{~cm}^{-3}$. Figure 4 exhibits the same qualitative deviations from an ideal photon echo as the experiment (Fig. 2). The echo width increases from $26 \mathrm{fs}\left(t_{21}=\right.$ $12.5 \mathrm{fs})$ to $50 \mathrm{fs}\left(t_{21}=87.5 \mathrm{fs}\right)$, which agrees qualitatively well with the experiment (Fig. 3). The echo maximum occurs earlier than the ideal echo for large $t_{21}$. This effect, however, is less pronounced than in the experiment. At yet larger $n_{\mathrm{eh}}$, which are not accessible experimentally here, the deviations from the ideal echo become also more pronounced in theory. An example of the timeintegrated FWM signal is shown by the full triangles in Fig. 1. Beside small structures, it decays according to $\propto e^{-t_{21} / \tau}$-in sharp contrast to previous theoretical work with a bare Coulomb potential, see, e.g., Refs. [15,16], which predicted a $\propto e^{-\alpha t_{21}^{3}}$ decay for the early-time and low-density regime. $\tau$ vs $n_{\text {eh }}$ is depicted in Fig. 5 together with the experimental data. In agreement with the early work of Shank et al. [1], we obtain a good fit with a power law according to $\tau^{-1}=\tau_{0}^{-1}+c n_{\mathrm{eh}}^{1 / 3} . \tau_{0}$ describes the density-independent scattering with LO phonons. In agreement with the experiment (insets in Fig. 1), the computed FWM spectra (inset in Fig. 5) indicate that, for different $n_{\mathrm{eh}}, \tau$ stems from different averages of optical band-to-band continuum transitions.

A bare Coulomb potential is not adequate for the description of the present FWM experiments, which becomes clear from a comparison with Ref. [15]. This shows that screening has already partially developed after several tens of femtoseconds under conditions where the inverse plasma frequency, $\omega_{\mathrm{pl}}^{-1}$, is longer than $50 \mathrm{fs}\left(n_{\mathrm{eh}}=\right.$ $\left.2.2 \times 10^{17} \mathrm{~cm}^{-3}\right)$. In contrast to this, previous pump/ probe experiments $[17,18]$ on bulk GaAs seemed to be less sensitive with respect to screening, so that these experiments could be described well with a bare Coulomb potential [18].

In conclusion we have observed qualitative deviations from an ideal photon echo response when exciting the band edge of GaAs with 11 fs pulses. These deviations are reproduced qualitatively by a quantum kinetic theory of carrier-carrier Coulomb scattering. Theory and experiment agree quantitatively in the famous power law of decay time versus carrier density $\tau^{-1}=\tau_{0}^{-1}+c n_{\mathrm{eh}}^{1 / 3}$. The deviations of the coherent response from an ideal echo, however, show that $\tau$ cannot simply be related to a single unique dephasing time $T_{2}$.

This work has been supported by the DFG-SPP Quantenkohärenz in Halbleitern and by the Krupp-Stiftung. One of us (Q.T. V.) gratefully acknowledges support by the KAAD. We thank W. Stolz for the high quality sample and M. U. Wehner who helped to build the setup.

[1] P. C. Becker et al., Phys. Rev. Lett. 61, 1647 (1988).

[2] J. Y. Bigot et al., Phys. Rev. Lett. 67, 636 (1991).

[3] A. Lohner et al., Phys. Rev. Lett. 71, 77 (1993).

[4] M. Lindberg et al., Phys. Rev. A 45, 1865 (1992).

[5] G. Noll et al., Phys. Rev. Lett. 64, 792 (1990).

[6] D. S. Kim et al., Phys. Rev. Lett. 68, 2838 (1992).

[7] D. S. Kim et al., Phys. Rev. Lett. 69, 2725 (1992).

[8] J.-Y. Bigot et al., Phys. Rev. Lett. 70, 3307 (1993).

[9] L. Bányai et al., Phys. Rev. Lett. 81, 882 (1998).

[10] L. Bányai et al., Phys. Rev. Lett. 75, 2188 (1995).

[11] M. U. Wehner et al., Phys. Rev. Lett. 80, 1992 (1998).

[12] H. Haug and J.-P. Jauho, Quantum Kinetics in Transport and Optics of Semiconductors, Springer Series in SolidState Sciences Vol. 123 (Springer-Verlag, Berlin, 1996).

[13] Q. T. Vu et al., Phys. Rev. B 59, 2760 (1999).

[14] Using self-consistently computed retarded Greens functions for both, Coulomb and LO-phonon scattering, and a carrier density of $n_{\mathrm{eh}} \approx 2 \times 10^{16} \mathrm{~cm}^{-3}$, the theory also reproduces the non-Markovian phonon oscillations discussed in Ref. [10].

[15] Q. T. Vu et al., Europhys. Lett. 40, 323 (1997).

[16] V. L. Gurevich et al., Europhys. Lett. 12, 4 (1990).

[17] S. Bar-Ad et al., Phys. Rev. Lett. 77, 3177 (1996).

[18] F. X. Camescasse et al., Phys. Rev. Lett. 77, 5429 (1996). 